\title{
Enhanced Segmentation Method for Bone Structure and Diaphysis Extraction from X-ray Images
}

\author{
N. Umadevi \\ HOD, Dept.of Computer Science \& IT, Sri Jayendra \\ Saraswathy Maha Vidyalaya College of Arts \& \\ Science, Coimbatore - 641005.
}

\author{
Dr. S.N Geethalakshmi \\ Associate Professor, Dept. of Computer Science, \\ Avinashilingam University for Women, \\ Coimbatore - 641043 .
}

\begin{abstract}
Medical imaging systems have been used in various medical application domains like trauma centre, orthopedic, pain management and vascular and non-vascular. One of the oldest and frequently used devices to capture human bones is X-Ray. During the process of identifying fractures, a vital step is the extraction of bone structure from the x-ray image. In this paper, a model that combines multi-resolution wavelets, region growing algorithm and active contour model is proposed to segment the bone structure from the $\mathrm{x}$-ray image. Further a fast Hough transformation is used to extract the diaphysis region from the segmented bone structure. Experimental results prove that the proposed algorithm is efficient both in the manner of segmentation and speed of segmentation.
\end{abstract}

\section{General Terms}

Region growing, Contour model, multi-resolution wavelets

\section{Keywords}

Bone Structure Extraction, Diaphysis Extraction, X-Rays

\section{INTRODUCTION}

Using advanced technology to increase the speed and accuracy of diagnosis in a trauma environment is the most frequently used application and helps in identifying fractures and sprains. An orthopedic surgeon could utilize these tools for alignment purposes as in hip and fracture pinning, thus saving time without having to reposition the patient or imaging device. The tools available today have made it possible to innovatively extract information about human body in a convenient and economical fashion. The continuing advances made available through both hardware and software demands new techniques and enhancement of existing techniques to be developed. It is a well-known fact that there is no common method that can be applied to analyze or process all parts of a human body and the techniques are dedicated to each part separately. Owing to this demand, this paper focuses on the bone part of human anatomy.

An x-ray makes images of any bone in the body and this paper considers only the leg bone of the human body. A leg bone is one of the longest bones in human body and consists of tibia and fibula (Figure 1). A tibia consists of diaphysis and Epiphysis. This paper considers the diaphysis part of tibia.

A typical ailment that affects the tibia is the fracture, which occurs when the bone is not able to withstand outside force like direct blows, twisting injuries and falls. Tibial fractures are the most common long bone fractures (Rockwood et al., 2006) and are a common affliction, accounting for approximately $20 \%$

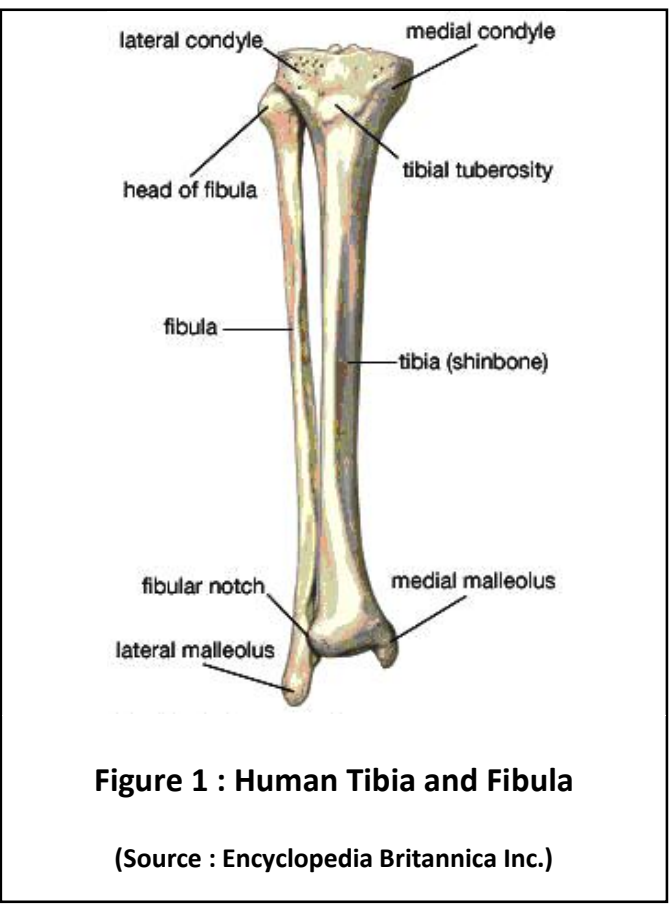

occupancy of hospitals wards at any given time (Chipchase et $a l ., 2000)$. Tibia bone fracture is one of the most common injuries encountered in clinical routine and trauma surgery and is still considered challenging as these injuries are different and variable in presentation and their outcomes are unpredictable. According to Poduval and Kale (2010) the incidence of tibial fractures has increased to approximately 500,000 cases in the United States per year. On average, almost 26 tibia fractures occur per 100,000 populations per year. This huge number of reported cases, in turn, is creating large number of X-ray images that has to be analyzed for diagnosis. It is a well-known fact that a tired or distracted person (doctor) will mis-diagnose a finding, stating something is "negative" when it was "positive" or vice versa (www.wrongdiagnosis.com/intro/common.htm). Further, often portions of x-ray images are obscured by lesions which overshadow subtle and makes a radiologist miss a fracture (Berbaum et al., 2001). As accurate diagnosis of fractures is vital to the effective management of patient injuries, the current need of the market is have tools that can automatically detect fractures from x-ray devices and can assist doctors as a automatic computer aided diagnosis system.

An Automatic Leg Bone Fracture Detection System (ALFDS) consists of four steps, namely, preprocessing, segmentation, fracture detection and location identification. Preprocessing consist of general image enhancement routines to improve the 
quality of the X-ray image and which will improve the accuracy of the subsequent steps. Segmentation is the process of extracting the Region of Interest (ROI) from the X-ray image. Fracture detection consists of steps to decide whether the presented image has normal or fractured bone. In case of fracture, the location identification step, locates the place of fracture and points it out to the physician. This paper focuses on developing a segmentation system to extract the ROI from the $\mathrm{X}$-ray device and is organized as follows. Section 2 presents the methodology used by the proposed segmentation algorithm. Section 3 presents the results obtained during performance evaluation. A conclusion with future research directions is presented in Section 4.

\section{RELATED STUDIES}

In comparison to the segmentation of images in other modalities, research in the field concerning X-ray images are less. Existing algorithms for the segmentation of X-ray images often rely on some general image segmentation methods. The general segmentation techniques can be grouped into threshold, region-based, edge-based, graph-based classification-based, deformable model and geometric methods. Apart from this, fuzzy techniques, watershed algorithms, wavelets have also been used.

While considering segmenting bone structure from X-ray images, in 1993, Manos et al. applied a region-based algorithm to segment hand and wrist bones. The algorithm starts with a region growing stage, followed by a region merging stage, which groups regions similarity, size, connectivity and edge information. The final segmentation result is obtained by region labeling according to some heuristic rules based on gray-level information. El-Feghi et al. (2004) applied a fuzzy set algorithm to segment lateral skull images. Three crisp subsets, namely background, skin and bones are determined by minimizing a fuzzy index function. The fuzzy index function decreases as the similarity between two pixels increases. As no spatial information is considered, the segmented bone regions are disjoint. McNitt-Gray et al. (1995) proposed to segment chest radiographs into anatomical regions using neural networks. 59 features including the gray level information, local difference measures and local texture measures are used as the input of the neural network. Spatial information is also used to correct misclassified pixels. Classification-based algorithms are generally not effective for $\mathrm{x}$-ray image segmentation due to the intrinsic properties of $\mathrm{x}$-ray images, i.e., images of different body parts overlap.

Vinhais and Campilho (2005) created a geometrical model of xray images by computing a mean shape from the training images. They applied a Laplacian of Gaussian (LoG) filter with high standard deviation on the image to extract some anatomical landmarks for an initial rough registration. They used genetic algorithm (GA) to find the coefficients of the geometric model in the free-form deformation stage. Each chromosome of GA contains an ordered list of the control points of a free-form deformation grid (Sederberg and Parry, 1986). Most of the $\mathrm{x}$-ray image segmentation work is based on deformable models, especially active contour model. In order to locate the rib border in chest radiographs, Yue et al. (1995) first determined the thoracic cage boundary to restrict the search space. Hough transform was then used to find approximate borders. The snake model was finally applied to refine the rib borders.

Jiang et al. (2004) used geodesic active contour (Caselles et al., $1995)$ to segment forearm bones. Geodesic active contour is based on the traditional snake model, and evolves over time according to geometric measures (curvatures) of the image. The initial snake contour is manually segmented from the $\mathrm{x}$-ray image of the patient at the initial visit to the hospital. Chen et al. (2005) proposed an incremental approach to segment leg bones. Salient features in the x-ray images including parallel lines in the shaft area, circles in the femoral heads are first detected. Then, a 2-D femur model is fitted onto the input $\mathrm{x}$-ray images to match the features. The final femur contours are refined by a snake algorithm with curvature constraints. To achieve this, a spring force which describes the difference between the actual curvature of the snake and the reference curvature of the model is introduced.

Similar work (leg bone segmentation) has been done by Behiels et al. (1999). The proposed algorithm is based on active shape model. The major contribution is that a regularization term representing the smoothness of shape change in each iteration is incorporated. Ballerini and Bocchi (2003) added an internal energy term to the snake framework to model the spatial relationships between adjacent bones to segment the long bones (hand). This energy term is represented by an elastic force that connects appropriate points of adjacent snakes. The snake model is represented in polar coordinates. The polar representation has the advantage that it introduces ordering of the contour points and prevents the snake elements from crossing each other during evolution. A semi-automatic approach was proposed by Bernard et al. (2001), which incorporated an articulated model during segmentation.

The solutions proposed for bone image segmentation can further be categorized into two main groups, namely, gray level feature-based methods and texture feature-based methods (Sharma and Aggarwal, 2010). Gray level feature-based methods analyze the gray level or color features of an image to segment an image. Histogram-based methods (Jung and Scharcanski, 2005), Edge-based methods (Law et al., 1996), region-based methods (Sonka et al., 1999) all belong to this category. Textural features of an image are considered important from both segmentation and classification point of view. The aim here is to divide images into regions with similar texture properties (Pavilidis, 1980). All these methods use random patterns/textures but work well for segmenting medical images. Apart from these, model based segmentation and Atlas based segmentation techniques have also been proposed. Model based methods involve active shape and appearance model during training. They work by determining and analyzing the statistical influence between the model and image during segmentation. These methods often require manual interaction and exhibits poor convergence to concave boundaries (Pham et al., 2000).

\section{EXISTING TECHNIQUES}

This paper combines region growing and active contours for segmenting the region of interest from the x-ray images. This section explains the basic concepts used by region growing and active contours models.

\subsection{Region Growing Algorithm}

Region growing is a technique for extracting a region of the image that is connected based on some predefined criteria like intensity information and/or edges in the image. In its simplest form, region growing requires a seed point that is manually selected by an operator and extracts all pixels connected to the initial seed with the same intensity value. Region Growing is an approach to image segmentation in which neighbouring pixels are examined and added to a region class if no edges are detected. This process is iterated for each boundary pixel in the 
region. If adjacent regions are found, a region-merging algorithm is used, in which, weak edges are dissolved and strong edges are left in tact. The traditional region based technique performs segmentation using the following three steps.

Step 1: Identifying a seed point within the bone structure of the $\mathrm{x}$-ray image.

Step 2: Calculating the connectivity of the points of the image to the seed point.

Step 3: Applying a selection criteria to the connectivity of the points (from Step 2 above) in order to identify the extent of the nodule (Halting criteria).

Region Growing offers several advantages. The borders of regions found by region growing are perfectly thin and well connected. The algorithm is very stable with respect to noise. Most importantly, membership in a region can be based on multiple criteria. It is possible to take advantage of several image properties, such as low gradient or gray level intensity value, at once, while using region growing algorithm.

\subsection{Active Contour Model}

There are two forms of contour models. The first is the parametric form and the second is the implicit form. In the parametric form, also referred to as snakes, an explicit parametric representation of the curve is used. This form is not only compact, but is robust to both image noise and boundary gaps as it constrains the extracted boundaries to be smooth. However, the performance depends on the correct selection of parameters. Moreover, when the topology or shape of an image changes, these parameters also has to be changed. This is difficult in real time environment. In contrast, the implicit deformable models, also called implicit active contours or level sets, are designed to handle topological changes naturally. In this paper, an implicit active contour for the segmentation of $\mathrm{x}$ ray images is considered and the traditional algorithm is explained in this section.

The user specifies an initial guess for the contour, which is then moved by image driven forces to the boundaries of the desired objects. In such models, two types of forces are considered - the internal forces, defined within the curve, are designed to keep the model smooth during the deformation process, while the external forces, which are computed from the underlying image data, are defined to move the model toward an object boundary or other desired features within the image.

The initial contour can be placed inside or outside an object and depending on this selection, it can move either inward or outward. If placed outside the object, the contour will segment the outer boundary of the object. But the disadvantage here is that it has an edge hole then the segmentation will not be correct. To solve this, the initial contour is placed inside the object. Here from the initial selection, the contour propagate outwards towards a region and can obtain the exterior boundaries of image object. Once the initial contour has been determined, we need to calculate the signed distance function, that is, the minimum distance from each pixel in the image to the prescribed initial contour. The Eikonal equation is used for this purpose. The Eikonal equation is a non-linear Partial Differential Equation (PDE) function. It finds the shortest time needed to travel from contour point to the boundary by using a time-cost function. Discretization concerns the process of transferring contour models and equations into discrete counterparts.

The main challenge while using active contour models for bone segmentation is the initial seed selection. Different initial seed values leads to different segmentation result and often incorrect selection produces inaccurate segmentation. To solve this problem, this paper proposes a model that uses the region growing algorithm to provide initial seeds which are then used by the active contour model. The proposed method further uses wavelets and morphological operators to enhance the segmentation process. The methodology used is explained in the following section.

\section{PROPOSED SCHEME}

The proposed segmentation scheme extracts the diaphysis using a two-step procedure. The first step 'Bone Structure Extractor (BSE)' identifies the bone structure from the x-ray image and the second step uses the resultant image of Step 1 to extract the diaphysis from the tibia bone structure. The BSE is designed as an amalgamation of image processing techniques. X-Ray images are often affected by Poisson noise which apart from reducing the visual quality also obscures important parts of the bone image. They often affect the diagnosis results and therefore have to be removed. For this purpose, the BSE algorithm uses a noise removal method which combines Independent Component Analysis with multiple wavelet denoising using enhanced Poisson Unbiased Risk Estimate (PURE) wavelet shrinkage method (Luisier et al., 2010) was utilized. More details on this algorithm can be found in (N.Umadevi and Dr.S.N.Geethalakshmi, 2011) is used. After enhancing the image, a multi-resolution wavelet transform is used to obtain multi-resolution representation of the input image. The motivation behind using multi-resolution wavelets is as follows. The bone structure in an $\mathrm{x}$-ray images are normally of different sizes. Wavelets have the ability to represent an image at different resolutions; each resolution characterizes different structures of the image. As the resolution gets coarser, it is possible to obtain general context image details of larger structures without complex details.

The wavelets use a hierarchical framework and each level passes through a low pass and high pass filter to capture the approximation and details of the image. After filtering, the image is subsampled by two, thus reducing the resolution by half. This decomposition step can be repeated using low-pass filtered subsamples as best approximate representation of the original image at multiple resolutions.. Let $2^{\mathrm{R}}$ represent the resolution of an image. The resolution of the original image can be obtained by assigning $\mathrm{R}=0$. Wavelets have the advantage that processing a single pixel at resolution $2^{\mathrm{R}}$ covers is equivalent to processing a block of size $2^{\mathrm{R}-1}$ pixels in the original image. Thus, processing a block $\mathrm{K}$ is equivalent to processing $2^{\mathrm{R}-1} \mathrm{~K}$ blocks. Therefore, the coarse information is analyzed over large neighborhoods whereas the detail information is analyzed over small neighborhoods.

The result of wavelet transformation is a set of images at different resolutions, which are segmented using an active contour segmentation. The problem of initial seed selection in active contour segmentation is solved by using region growing algorithm. The region growing algorithm used for preliminary segmentation uses the colour information of the image. The steps are given below.

1. Convert the RGB (Red, Green, Blue) colour space is converted to a HIS (Hue, Saturation, Intensity) colour space (Gonzalez et al., 2003). 
For each resolution image, determine the maximum and minimum intensity (I Component) and calculate its mean value. Also calculate the standard deviation. Let this be denoted as $\mu$ and $\sigma$ respectively.

3. Calculate the threshold $\mathrm{T}$ as $\sigma+0.2 \mu$. The value 0.2 was obtained after performing several experiments and selecting the one which gave optimum result.

4. Mark regions less than $\mathrm{T}$ as background and the rest as foreground regions.

The result of the region growing method is then used as initial seeds by the active contour model. The procedure used is given below.

1. Obtain initial contours from region growing algorithm

2. Set the contour shape as circle and obtain the initial assumed distance.

3. At the beginning of each iteration calculate external energy $\left(\mathrm{E}_{\text {ext }}\right)$ with respect to $\mathrm{x}$ and $\mathrm{y}$ separately.

4. Call level set algorithm for all $\mathrm{N}$ iterations do

Find shortest traveling time from a point $\mathrm{x}$ to boundary

Estimate Energy $\left(\mathrm{E}_{\text {ext }}\right)$ and calculate Partial Differential Equation

Determine distance, curvature terms, gradient, speed

Update level set function

if Iterations $\leq \mathrm{N}$ then

Reinitialize seeds

Calculate change and record in Contour

end

end

5. Display Segmented image

The result of the above algorithm is the separated bone structure from the x-ray image. This result is then segmented again to identify the diaphysis region. The approach used for this purpose is the same as Donnelley et al (2008). In this method, a Hough transformation is used to identify peaks. In the proposed framework, the traditional Hough algorithm is replaced by a fast Hough transformation (Hari et al., 2009) to optimize the speed of the algorithm. This algorithm modifies the standard Hough algorithm by considering a pair of pixels simultaneously and mapping them to the parameter space. The enhanced Hough algorithm is used to estimate the peak spread from the bone structure image. This estimation along with two thresholds is used to the detect parameterized Threshold one is used to retain only those regions where the line matches the bone edge and the threshold two is used to select only those lines with minimum length. The end points or beginning of diaphysis region is located by identifying edges that deviate from the line. A bone center-line is detected by analyzing the Hough peak lines. All lines that are close to each other are paired so that the line endpoints are relatively close to each other. The criterion for determining the correct pairing is to select the minimum total distance between the line endpoints. Thus, all possible pairings were analyzed and the combination that minimized this distance is selected as centre line. When multiple center-lines are detected, the one with that is wide along the axis of the bone was retained and the resulting bone centre line. The two-step process of the proposed method reduces the time complexity of automated fracture detection system by removing regions that that do not need attention during diaphysis fracture detection. Further, the actual process of segmentation is easy to implement and simplifies tedious tasks. The algorithm is consolidated in Figure 2 .

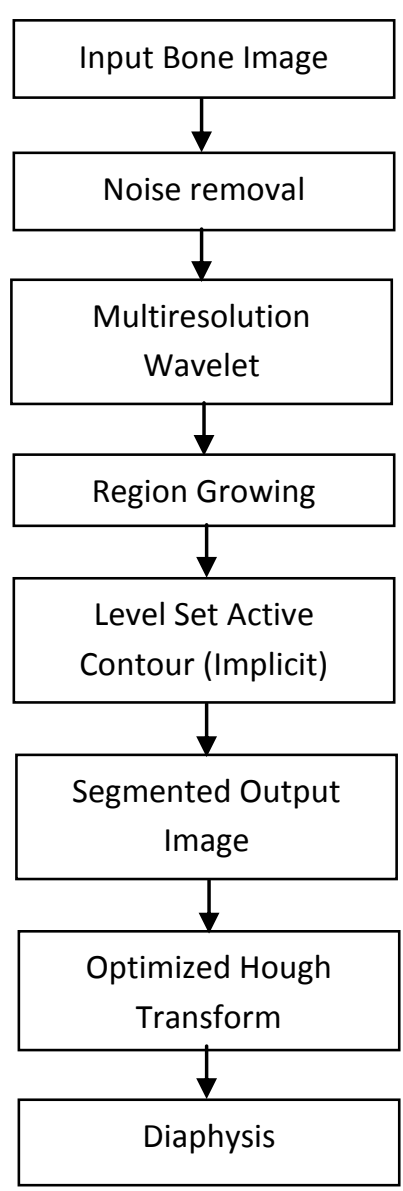

Figure 2 : Proposed Scheme

\section{EXPERIMENTAL RESULTS}

Several experiments were conducted to analyze the performance of the proposed segmentation algorithm. The experiments were conducted on a dataset containing $50 \mathrm{x}$-rays images containing both fractured and normal bones. The segmentation model was developed in MATLAB 2009a and was tested on a Pentium IV machine with 4 GB RAM. Figure 3 shows the results of four randomly selected images. From the visual results, it could be seen that the proposed BSE algorithm produces better segmentation results than the traditional region growing and active contour algorithms.

Execution speed is another performance metric that is used to analyze the performance of the algorithms. It is defined as the 
time taken by the algorithm to produce the segmented image. The results obtained are shown in Table 1. For better comparison all the images were resized to $256 \times 256$ pixels and the projected results does not take diaphysis segmentation into account.

From the Table 1, it is evident that the BSE algorithm produces quick result when compared to the traditional region growing and active contour segmentation modes. Thus, the experimental results show that the BSE algorithm is an improved version to both the traditional algorithms and can be used to segment bones from x-ray images. These promising results further prove that it can be used by any computer aided fracture detection systems.

\begin{tabular}{|c|c|c|c|}
\hline Image & Region & Contour & BSE \\
\hline Image1 & 14.62 & 15.98 & 13.64 \\
\hline Image2 & 13.56 & 15.26 & 13.16 \\
\hline Image3 & 14.81 & 16.18 & 13.99 \\
\hline
\end{tabular}

Table 1 : Segmentation Time (Seconds)

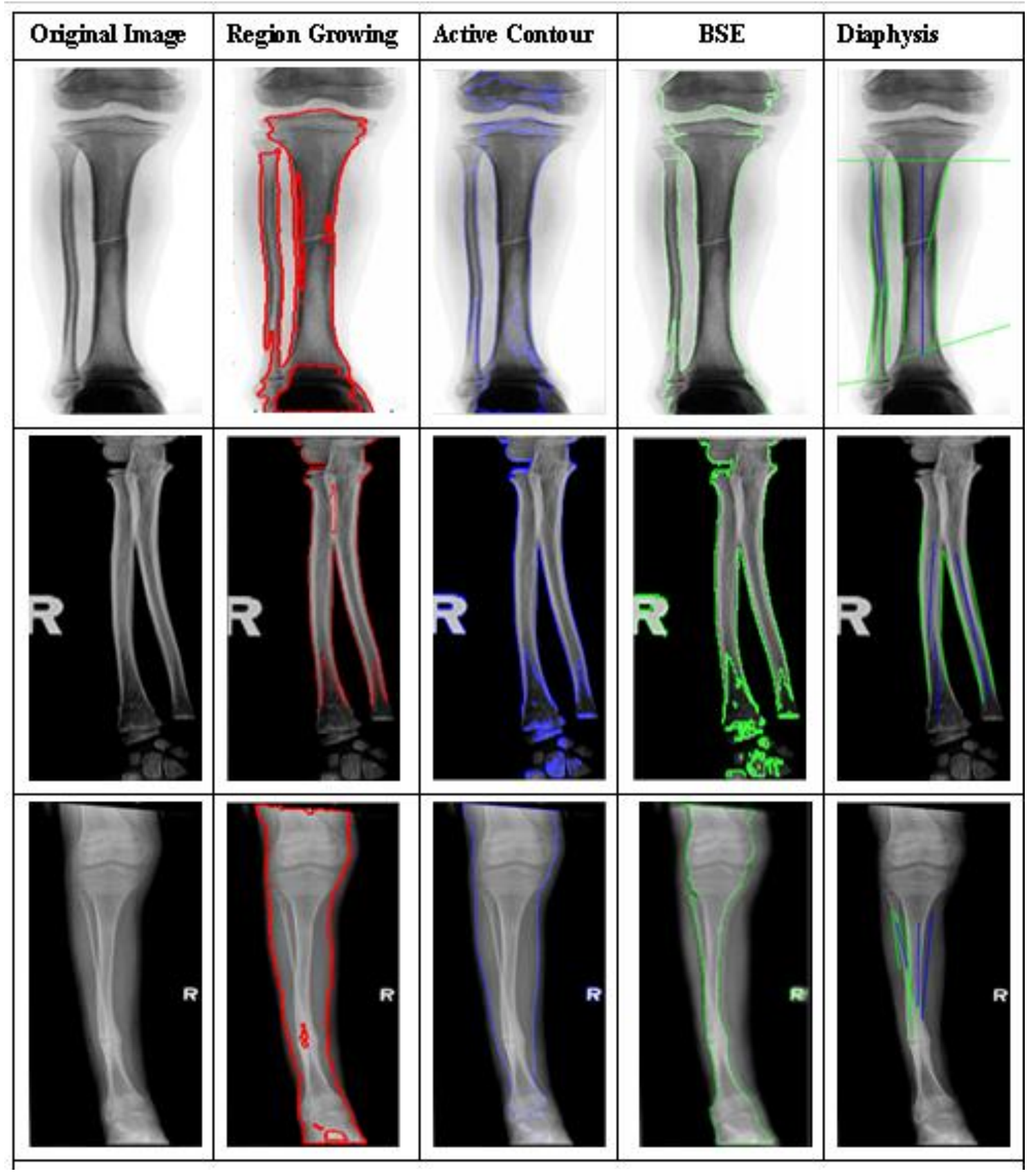

Figure 3 : A Visual Comparison 


\section{CONCLUSION}

This paper introduced a segmentation model that segments the bone structure and diaphysis region from a x-ray image. The BSE algorithm used wavelet decomposition, region growing algorithm and active contour model in a serial fashion to extract the bone structure from the $\mathrm{x}$-ray image. A fast Hough transformation was used to detect the diaphysis region. The experimental results prove that the proposed algorithm has significant gain in terms of segmentation accuracy and speed. In future, this algorithm will be combined with a fracture detection algorithm to detect and locate the region of fracture.

\section{REFERENCES}

[1] Aksoy, S. and Akcay, H.G. 2005, Multi-resolution Segmentation and Shape Analysis for Remote Sensing Image Classification, Proceedings of 2nd International Conference on Recent Advances in Space Technologies, pp. 599 - 604 .

[2] Ballerini, L. and Bocchi, L. 2003, Bone segmentation using multiple communicating snakes, SPIE International Symposium Medical Imaging.

[3] Behiels, G., Vandermeulen, D., Maes, F., Suetens, P. and Dewaele, P. 1999, Active shape model-based segmentation of digital x-ray images, Proceedings of the Second International Conference on Medical Image Computing and Computer-Assisted Intervention, Pp. 128-137.

[4] Berbaum, K. S., Brandser, E. A., Franken, J.E. A., Dorfman, D. D., Caldwell, R. T. and Krupinski, E. A. 2001, Gaze dwell times on acute trauma injuries missed because of satisfaction of search, Academic Radiology, Vol. 8, No. 4, Pp. $304-314,2001$.

[5] Bernard, R., Likar, B. and Pernus, F. 2001, Segmenting articulated structures by hierarchical statistical modeling of shape, appearance and topology, Medical Image Computing and Computer Assisted Intervention - 4th MICCAI, Pp. 499-506.

[6] Caselles, V., Kimmel, R. and Sapiro, G. 1995, Geodesic active contours, ICCV, pp. 694-699.

[7] Chen, Y., Ee, X., Leow, W. K. and Howe, T. S. 2005 , Automatic extraction of femur contours from hip x-ray images, Proc. First International Workshop on Computer Vision for Biomedical Image Applications (CVBIA 2005, pp. 200-209.

[8] Chipchase, L.S., McCaul, K. and Hearn, T.C. 2000, Hip fracture rates in South Australia: Into the next century, Australian and New Zealand Journal of Surgery, Vol. 70, No. 2, pp. 117-119.

[9] Donnelley, M., Knowles, G. and Hearn, T. 2008, A CAD System for Long-Bone Segmentation and Fracture Detection, Image and Signal Processing, Lecture Notes in Computer Science, Vol. 5099/2008, pp. 153-162.

[10] El-Feghi, I., Huang, M.A. and Ahmadi, M. 2004, X-ray image segmentation using auto adaptive fuzzy index measure, 2004 47th Midwest Symposium on Circuits and Systems, Vol. 3, 2004, pp. 499-502.

[11] Gonzalez, R.C., Woods, R.E. and Eddins, S.L. 2003, Digital Image Processing Using MATLAB, PrenticeHall, Inc.
[12] Hari C.V., Joseph, J.V., Gopi, S., Felix V.P. and Amudha J. 2009, Mid-Point Hough Transform: A Fast Line Detection Method, 2009 Annual IEEE India Conference (INDICON 2009), pp. 217-220.

[13] Jiang, Y. and Babyn, P. 2004, X-ray bone fracture segmentation by incorporating global shape model priors into geodesic active contours, Proceedings of the 18th International Congress and Exhibition, Vol. 1268, pp. 219-224.

[14] Jung, C.R. and Scharcanski, J. 2005, Robust watershed segmentation using wavelets, Image and Vision Computing, Vol. 23, Issue 7, pp. 661-669.

[15] Law, T., Itoh, H. and Seki, H. 1996, Image filtering, edge detection, and edge tracing using fuzzy reasoning, IEEE Trans PAMI, Vol. 18, pp.481-491.

[16] Luisier, F., Vonesch, C., Blu, T. and Unser, M. 2010 , Fast interscale wavelet denoising of Poisson-corrupted images, signal Processing, Elsevier, pp. 415-427.

[17] Mallat, S. 1996, Wavelets for a vision, Proceedings of the IEEE, Vol. 84, No. 4, pp. 604-614.

[18] Mallat, S.G. 1989, A theory for multiresolution signal decomposition: The wavelet representation, IEEE Transactions on Pattern Analysis and Machine Intelligence, Vol. 11, No. 7, pp. 674-693.

[19] Manos, G. K., Cairns, A. Y., Rickets, I. W. and Sinclair, D. 1993, Segmenting radiographs of the hand and wrist, Computer Methods and Programs in Biomedicine, Vol. 43, No. 3-4, pp. 227-237.

[20] McNitt-Gray, M. F., Huang, H. K. and Sayre, J. W. 1995, Feature selection in the pattern classification problem of digital chest radiograph segmentation, IEEE Transactions on Medical Imaging, Vol. 14, No. 3, pp. 537-547.

[21] Pavilidis, T. 1980, Structural description and graph grammar, Chang SK, Fu KS, editors. Pictorial information systems, Springer Verlag Berlin, pp. 86103.

[22] Pham, D.L., Xu, C. and Prince, J.L. 2000, Current methods in medical image segmentation. Ann Rev Biomed Engg., Vol. 2, pp. 315-37.

[23] Poduval, M. and Kale, S. 2010, Diaphyseal tibial fractures, Medscape Article, http://emedicine.medscape.com/ article/1248857overview, Last Access of August, 2011.

[24] Rockwood, C.A., Green, D.P. and Bucholz, R.W. 2006, Rockwood and Green's fractures in adults, 6th ed., Vol. 2, Lippincott Williams \& Wilkins, Philadelphia.

[25] Sederberg, T. W. and Parry, S. R. 1986, Free-form deformation of solid geometric models, ACM SIGGRAPH Computer Graphics, Vol. 20, 1986, pp. 151-160.

[26] Sharma, N. and Aggarwal, L.M. 2010, Automated medical image segmentation techniques, J Med Phys,Vol.35, pp.3-14.

[27] Sonka, M., Hlavac, V. and Boyle, R. 1999, Image processing, analysis and machine vision, Singapore: Thomson Learning. 
[28] Tamisiea D.F. 2008, Radiologic aspects of orthopedic diseases, Mercier LR, ed. Practical Orthopedics, 6th ed. Philadelphia, Pa: Mosby Elsevier; Chap 16.

[29] Umadevi N and Dr.Geethalakshmi S.N.,2011, Improved hybrid model for denoising poisson corrupted X-ray images, IJCSE,Vol. 3, pp. 2610-2619.

[30] Vinhais, C. and Campilho, A. 2005, Genetic modelbased segmentation of chest $\mathrm{x}$-ray images using free form deformations, Image Analysis and Recognition: Second International Conference, ICIAR 2005.

[31] How Common is Misdiagnosis? Available at: www.wrongdiagnosis.com/intro/common.htm

[32] Yue, Z., Goshtasby, A. and Ackerman, L. V. 1995, Automatic detection of rib borders in chest radiographs, IEEE Transactions on Medical Imaging, vol. 14, no. 3, pp. 525-536.

\section{AUTHORS BIOGRAPHY}

Dr. S.N. Geethalakshmi working as Reader in the Department of Computer Science, Avinashilingam University for
Women. She has nineteen years of teaching experience and ten years of research experience. Her area of interest is Image Processing, Software engineering. Her publications include 3 International journals, 3 National Journals, 9 International Conferences, 3 National Conferences and 2 National Articles.

N. Umadevi working as Head in the Department of Computer Science and Information Technology cum NSS Programme officer, Sri Jayendra Saraswathy Maha Vidyalaya College of Arts and Science, Singanallur,Coimbatore is a part time Research Scholar of Avinashilingam University for Women, Coimbatore doing her research under the guidance of Dr.S.N.Geethalkshmi. The Scholar has 3 years of industrial experience and 9 years of teaching experience. Her area of interest are Image Processing and Data Mining. Her publications include 3 International Journals. She has presented in 3 International Conferences and 3 national conferences. 